\title{
High Electrical Conductivity and Conspicuous Phase Transitions in Single Crystals of K-TCNQ
}

\author{
Yadunath Singh \\ Department of Physics, College of Engineering and Technology, Bikaner, India \\ Email:singh_kyn@yahoo.co.in
}

How to cite this paper: Singh, Y. (2017) High Electrical Conductivity and Conspicuous Phase Transitions in Single Crystals of K-TCNQ. Advances in Materials Physics and Chemistry, 7, 375-394.

https://doi.org/10.4236/ampc.2017.711030

Received: October 20, 2017

Accepted: November 27, 2017

Published: November 30, 2017

Copyright $\odot 2017$ by author and Scientific Research Publishing Inc. This work is licensed under the Creative Commons Attribution International License (CC BY 4.0).

http://creativecommons.org/licenses/by/4.0/

\section{cc) (i) Open Access}

\begin{abstract}
This paper is to report the temperature dependent electrical conductivity of single crystals of radical ion salt (RIS) potassium-TCNQ (K-tetracyanoquinodimethane) in a wide range of temperatures from 30 to $500 \mathrm{~K}$. This RIS is quasi-one-dimensional in nature. These single crystals of K-TCNQ are grown by different methods like electrochemical, solution growth and diffusion method. Activation energy is determined for the sample in different temperature regions and found different values. More than one semiconductor to metal phase transition is observed in the studied samples during electrical measurements below and above room temperature. All the features observed in the studied samples are analyzed in the framework of their molecular structure as well as under different effects like disorder, impurity, Coulomb interaction, charge density wave (CDW), scattering and 3-D effects etc.
\end{abstract}

\section{Keywords}

Electrical Conductivity, Radical Ion Salt, Tetracyanoquinodimethane, Charge Density Wave, Charge Transfer Complexes, A Semiconductor to a Metal Phase Transition

\section{Introduction}

The tremendous utility of highly conducting materials [1] [2] justifies the intense research in this domain. Charge transfer complexes $\left(\mathrm{CTC}_{\mathrm{s}}\right)$ or radical ion salts $\left(\mathrm{RIS}_{\mathrm{s}}\right.$ ) obtained from tetrathiafulvalene (TTF) or its analogs and various electron acceptors, such as tetracyanoquinodimethane (TCNQ), are considered good models for the development of organic conductors [3] [4] [5]. Hundreds of different molecular complexes containing TCNQ as the acceptor have been made [6] [7] [8] [9]. Due to the presence of the four cyno groups on the molecules and the relatively large $\pi$ conjugation system, reduction of the radical 
anion occurs very easily in TCNQ. This makes TCNQ as a very good electron acceptor. In result, TCNQ forms stable solid complexes containing both neutral $\mathrm{TCNQ}^{\circ}$ and anion $\mathrm{TCNQ}^{-}$with a wide variety of electron donating compounds. It has the ability to form fusible conductive materials and magnetically ordered structures, including ferromagnetic ones with high Curie temperatures, spin ladders and quasi-two-dimensional organic conductors and superconductors.

Because of their special structural features, these complexes exhibit a wide range of electrical conductivity at room temperature. TCNQ salts with metal cations generally show low to intermediate conductivities $\left(10^{-5}\right.$ to $\left.10^{-2} \Omega^{-1} \mathrm{~cm}^{-1}\right)$, while its salts with cations of plane aromatic heterocyclic molecules show reasonably higher conductivities $\left(\approx 10^{2} \Omega^{-1} \mathrm{~cm}^{-1}\right)$. Some investigations have been made on the electrical conduction of TCNQ salts with metal cations so far [10] [11]. In these alkali metal salts [12], a phase change has also been reported in the temperature range from 120 to $500 \mathrm{~K}$.

These TCNQ-complexes have drawn intensive interest due to their unique physical properties [13] and widely potential applications in organic nano-devices and other fields also. Due to their electrical nonlinearity and switching behavior, these are quite suitable for making nonvolatile memories [14] [15] [16]. The TCNQ complexes can be used as ultra-high density data storage via thermochemical hole burning using a scanning tunneling microscope (STM) [17] [18], organic metal electrodes in inkjet printing [19] and organic light emitting devices [20] [21]. High-performance low-cost organic field-effect nano-transistors [22] [23] and field emission devices [24] based on charge-transfer crystals of TTF-TCNQ, Cu-TCNQ, Ag-TCNQ were also investigated.

Some RIS in the TCNQ system exhibit characteristics such as anisotropy, electronic and structural instabilities. For instance, their electrical conductivity and electron spin resonance spectra were highly anisotropic with respect to the crystal axis [6] [25] [26]. Metal-insulator transition has reported in many TCNQ complexes such as TTF-TCNQ [27] [28], NMe ${ }_{3} \mathrm{H}-\mathrm{I}-\mathrm{TCNQ}$ [26], BEDT-TTF-TCNQ [29], (BETS) ${ }_{2}-\mathrm{Br}_{2}$-TCNQ [30] and CPDT-STF-TCNQ [31]. Other phase transitions such as neutral-ionic phase transition in TTF-CA [32] [33] [34] [35] and TMB-TCNQ [33] semiconductor to semiconductor transition in $\mathrm{NMe}_{3} \mathrm{H}-\mathrm{I}-\mathrm{TCNQ}$ [26] and spin-Peierls transition in MEM-TCNQ) ${ }_{2}$ [36] were also reported.

In recent years a large number of new charge-transfer complexes have been synthesized and characterized and their physical properties need to be further investigated. Some of those are still interesting and attractive, such as enantiotropic solid-state phase transition [37] electronic and structural instabilities of mixed-stack organic charge transfer complexes [35] etc.

Although Bloch et al. [38], Kamra et al. [39] and Cowan et al. [40] have done pioneering work about the effect of temperature on the conductivity of some organic radical ion salts (RIS). In most of the samples, general features of temperature dependence conductivity are same. In the low-temperature region, be- 
low about $50 \mathrm{~K}$, conductivity falls rapidly or gradually, depending upon the nature of the material studied. Conductivity, in general increases with some orders in higher temperature region. This paper reports the synthesis of K-TCNQ samples by using three methods. The temperature dependent DC electrical conductivity was measured in a wide range of temperature from 30 to $500 \mathrm{~K}$ on all the grown samples. A semiconducting behavior was observed at room temperature with the marked difference in electrical conductivity and activation energy. The electrical conductivity and activation energy measured at room temperature in this study is 3 orders more and one order less respectively than the reported earlier. These obtained results for the K-TCNQ are compared also with previously reported studies in Table 1.

In particular, some conspicuous phase transitions occur at different temperatures at below and above the room temperature. Those are different from previously reported transitions temperatures. This nonlinear and switching behavior of the studied complex make it more interesting and increase its potential and applicability in making nonvolatile memories, ultra-high density data storage and organic metal electrodes in inkjet printing. A change of one order less in activation energy also make it quite suitable material for development of light emitting devices, organic field-effect nano-transistor and field emission devices.

\section{Experiments}

\subsection{Synthesis and Characterization}

The single crystals of K-TCNQ were grown by three methods like as Solution growth [6], diffusion method and electrochemical methods [41]. In all cases the potassium iodide and TCNQ were purified by re-crystallization in acetonitrile at $100^{\circ} \mathrm{C}$ and always used in 1:1 composition to grow these complexes.

\subsubsection{Solution Growth Method}

The purified potassium iodide and TCNQ were separately dissolved in acetonitrile in 1:1 ratio composition. Both of the solutions are heated separately and boiling solution of potassium iodide is slowly added to the boiling solution of TCNQ. After a slow evaporation at the room temperature, small cubical shaped blackish crystals of typical dimensions were obtained.

\subsubsection{Diffusion Method}

The diffusion method was also used to grow large single crystals of charge transfer complexes. Here in this experiment, diffusion method as described by Kaplan [42] and Anzai [43], was used to grow potassium-TCNQ single crystals. It provides very good results in the case of purity and size of the crystals.

\subsubsection{Electrochemical Method}

Although the conventional solution growth method is the easiest one to grow crystals but the size and purity were not found satisfactory in several previous experiments. Therefore, electrochemical method [41] was also used to grow this 
Table 1. A comparative study of electrical conductivity at room temperature, transition temperatures and activation energy of K-TCNQ single crystals reported in earlier studies and this paper.

\begin{tabular}{|c|c|c|c|c|c|}
\hline Synthesis Method & $\begin{array}{c}\sigma_{R T} \\
\left(\Omega^{-1} \mathrm{~cm}^{-}\right)\end{array}$ & $\begin{array}{c}\text { Transition } \\
\text { Temperature } K\end{array}$ & $\Delta E(\mathrm{eV})$ & $\begin{array}{c}\text { Temperature } \\
\Delta \mathrm{E} \text { determined }\end{array}$ & References \\
\hline Solution Growth & $3.5 \times 10^{-4}$ & 395 & 0.43 & Room temperature & 54 \\
\hline Diffusion Method ${ }^{\mathrm{b})}$ & $1.0 \times 10^{-4}$ & 400 & 0.42 & Room temperature & 55 \\
\hline Solution Growth ${ }^{c)}$ & - & 381 & - & - & 12 \\
\hline Solution Growth ${ }^{\mathrm{d})}$ & $2.0 \times 10^{-4}$ & - & - & - & 65 \\
\hline Solution Growth ${ }^{\mathrm{e}}$ & $5.0 \times 10^{-7}$ & 373 & 0.43 & Room temperature & 11 \\
\hline Diffusion Method ${ }^{\mathrm{f}}$ & $1.0 \times 10^{-4}$ & 391.6 & 0.45 & Room temperature & 56 \\
\hline \multirow[t]{2}{*}{ Solution Growth ${ }^{\mathrm{g})}$} & $1.0 \times 10^{-4}$ & 394 & - & - & 66 \\
\hline & & 120 & 0.1 & $\begin{array}{c}\text { Room temperature } \\
(300)\end{array}$ & Our results \\
\hline \multirow[t]{4}{*}{ Solution Growth ${ }^{\mathrm{h}}$} & $2.4 \times 10^{-4}$ & 270 & 0.078 & $50-100$ & \\
\hline & & 380 & 0.032 & $265-270$ & \\
\hline & & 470 & 0.13 & $380-450$ & \\
\hline & & 120 & 0.09 & $\begin{array}{c}\text { Room temperature } \\
(300)\end{array}$ & \\
\hline \multirow[t]{5}{*}{ Diffusion Method ${ }^{\mathrm{h}}$} & $2.5 \times 10^{-4}$ & 270 & 0.076 & $50-100$ & Our results \\
\hline & & 380 & 0.030 & $265-270$ & \\
\hline & & 470 & 0.12 & $380-450$ & \\
\hline & & & 0.06 & $\begin{array}{l}\text { Room temperature } \\
(300)\end{array}$ & \\
\hline & & & 0.086 & $50-100$ & \\
\hline \multirow[t]{3}{*}{ Electrochemical $^{\text {h) }}$} & $7.0 \times 10^{-1}$ & 270 & 0.069 & $265-270$ & Our results \\
\hline & & & 0.048 & $380-450$ & \\
\hline & & & 0.12 & Above 450 & \\
\hline
\end{tabular}

${ }^{\mathrm{a}}$ Reference [54]; ${ }^{\mathrm{b}}$ Reference [55]; ${ }^{\mathrm{C}}$ Reference [12]; ${ }^{\mathrm{d}}$ Reference [65]; ${ }^{\mathrm{e}}$ Reference [11]; ${ }^{\mathrm{f}}$ Reference [56]; ${ }^{\mathrm{g}}$ Reference [66]; ${ }^{\text {hPresent study. }}$

salt. In this method, long needle-shaped crystals of K-TCNQ at the electrodes with stoichiometry $1: 1$ were obtained.

The obtained crystals are larger in size and highly electrically conducting. The grown crystals were characterized by usual methods like elemental analysis, atomic spectra, infra-red spectra, NMR patterns and x-ray diffraction patterns etc. All the obtained results in characterization were well matched and also in confirmation with previously reported studies of the complex.

\subsection{Electrical Conductivity Measurement}

These measurements were carried out at normal pressure by four probe method [44] with a Keithley constant current source, digital electrometer, and Keithley null detector micro-voltmeter. Prior to measurement, the flat surfaces of the 
samples were contacted with the probes using silver paste to ensure good electrical contacts. To minimize the adsorption of gases on the samples, a suitable sample holder was fitted inside to a high vacuum chamber of liquid helium close cycle refrigerator.

The temperature of the samples was measured at two ends with the help of two temperature sensors, placed at lower and upper surface of the samples. The temperature of the sample was controlled, stabilized and gradually changes with the help of Lakeshore temperature controller. The measurements were performed in such a way that the temperature of the samples was held uniform. A lot of care was taken to avoid the undesirable temperature gradient.

Here, it is notable that some difficulties arose during the measurement of the electrical conductivity with temperature variation in case of the single crystals. In most of the cases crystals are small, thin and fragile, so a great care must be taken in sample mounting as well as a change in temperature of the mounted sample. In case of highly conducting crystals, one has to avoid large contact resistance, so the four probe method must be used. The available crystals are small in size and Joule heating of the sample may occur at relatively low power; hence large current should not be allowed to pass through the crystals.

Temperature dependence electrical conductance was observed in every single crystal separately grown by electrochemical, diffusion and solution growth methods as discussed below:

\subsubsection{Single Crystal Grown by Electrochemical Method}

The electrical conductivity of needle-shaped, purple-bluish single crystal of K-TCNQ grown by electrochemical method [41] is found to be $\sigma_{R T} \approx 7 \times 10^{-1}$ $\Omega^{-1} \mathrm{~cm}^{-1}$ at room temperature $(300 \mathrm{~K})$ with an activation energy of $0.06 \mathrm{eV}$. The observed electrical conductance behavior of this sample in the temperature range 30 to $500 \mathrm{~K}$ is plotted and shown in Figure 1(a) and Figure 1(b). It is clear from these figures that the conductivity first increases with a decrease in temperature up to $270 \mathrm{~K}$ and then starts decreasing. The drop in conductivity is much faster below the temperature $260 \mathrm{~K}$ as evident from Figure 1(b). A sharp fall of the order of $10^{-2}$ magnitude in conductivity is also observed around the temperature $272 \mathrm{~K}$. Above the room temperature, the conductivity of the complex initially decreases and reaches a minimum at $380 \mathrm{~K}$. Above to this temperature, it starts to increase and change by an order of magnitude in the temperature range 380 to $500 \mathrm{~K}$.

On theoretical fitting of this behavior, it is observed that conductivity follows the law $T^{2}$ in metallic region $270 \leq T \leq 300 \mathrm{~K}$, below the room temperature. The semiconducting nature of the sample is well fitted by usual exponential law as $\ln \sigma$ vs $T^{-1}$ in different temperature regions as shown in the Figure 4 and Figure 5. This nature of the sample is observed in the region $200 \leq T \leq 270 \mathrm{~K}$ with an activation energy of $0.069 \mathrm{eV}$ and $160 \leq T \leq 190 \mathrm{~K}$ with an activation energy of $0.086 \mathrm{eV}$. Above the room temperature, metallic nature of the sample is well fitted with $T^{2}$ and $T^{-1}$ laws in temperature region $300 \leq T \leq 345 \mathrm{~K}$ and $345 \leq T \leq$ 


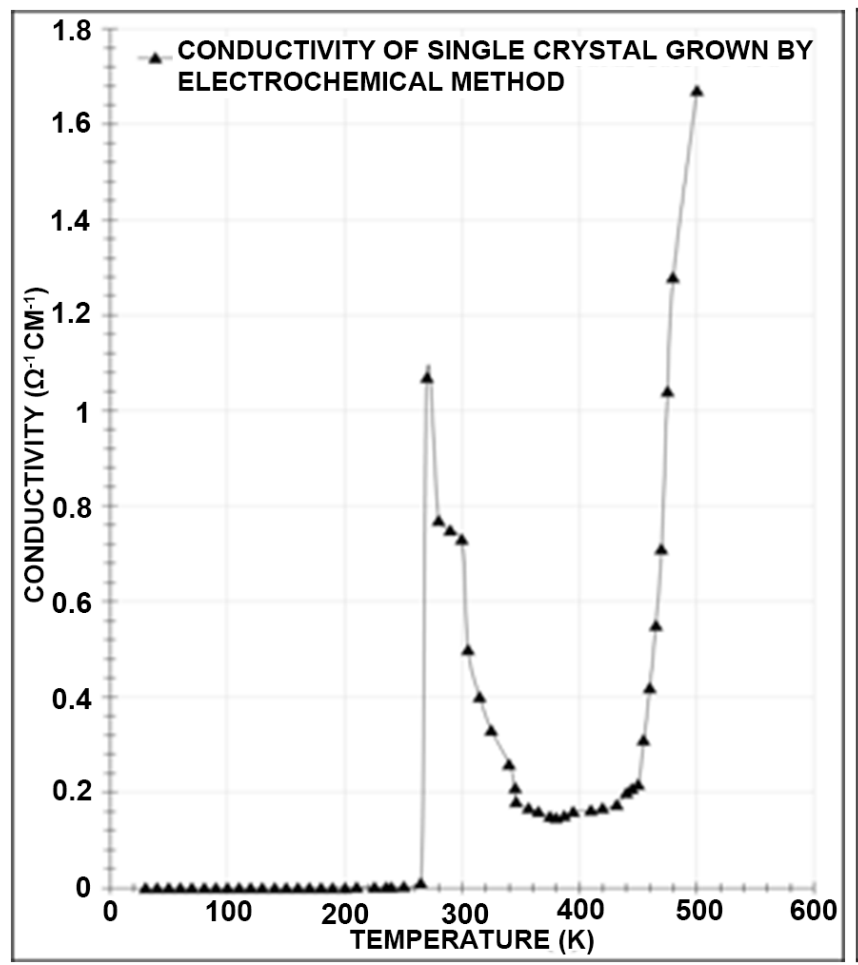

(a)

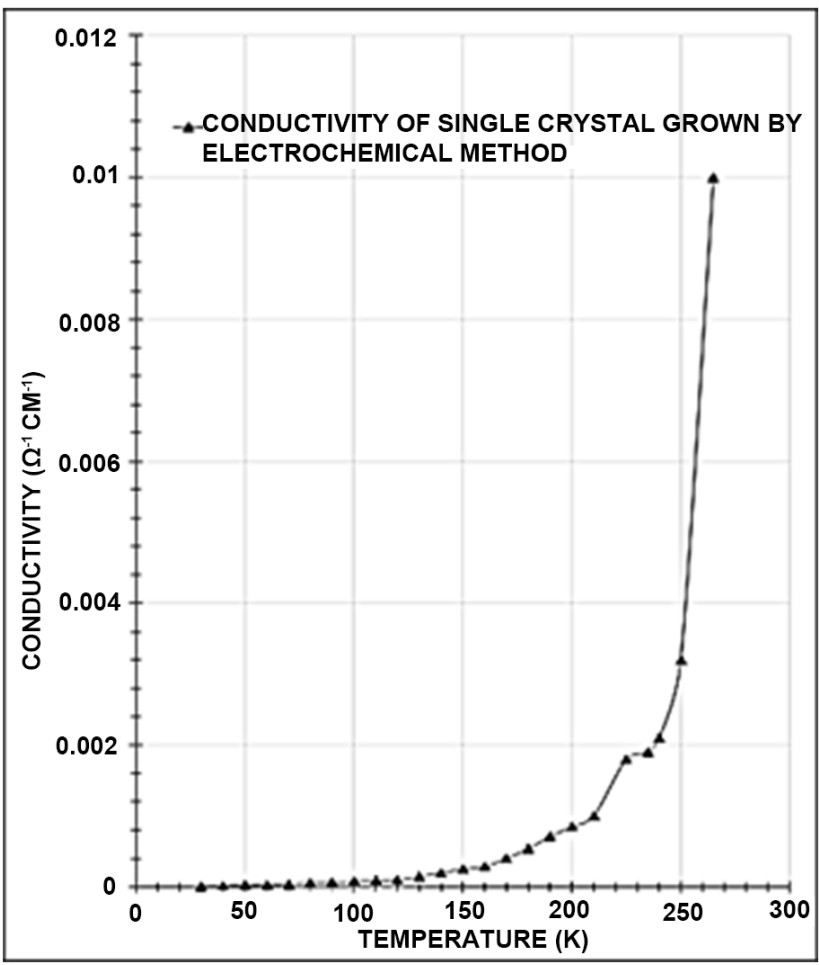

(b)

Figure 1. (a) Plot of conductivity as a function of temperature in the region 30 to $500 \mathrm{~K}$ for K-TCNQ single crystal grown by electrochemical method; (b) Plot of conductivity as a function of temperature in the region 30 to $265 \mathrm{~K}$ for K-TCNQ single crystal grown by electrochemical method.

$380 \mathrm{~K}$ respectively. Whereas semiconducting nature of the sample above room temperature, which obeys the relation $\ln \sigma$ vs $T^{-1}$ in temperature region above $380 \mathrm{~K}$. The activation energy was calculated in this region and a value of 0.048 $\mathrm{eV}$ in $380 \leq T \leq 450 \mathrm{~K}$ and $0.12 \mathrm{eV}$ above the $450 \mathrm{~K}$ was obtained.

\subsubsection{Single Crystal Grown by Solution Growth Method}

Blackish, cubical shaped single crystal of K-TCNQ grown by solution growth method in acetonitrile have shown electrical conductivity $\sigma_{R T} \approx 2.4 \times 10^{-4}$ $\Omega^{-1} \mathrm{~cm}^{-1}$ at room temperature with an activation energy of $0.1 \mathrm{eV}$. This sample shows a typical behavior of conductivity with variation in temperature in the range of 30 to $500 \mathrm{~K}$. On starting, the measurement with increasing the temperature from $30 \mathrm{~K}$, initially the conductivity increases. It reaches a maximum value of conductivity $1.48 \times 10^{-3} \Omega^{-1} \mathrm{~cm}^{-1}$ at a temperature $120 \mathrm{~K}$ with a change of the magnitude of an order 10 . On further increasing the temperature, conductivity goes down and reaches a value $4.2 \times 10^{-4} \Omega^{-1} \mathrm{~cm}^{-1}$ at a temperature $265 \mathrm{~K}$. On further increase in the temperature, the conductivity of the complex starts increasing again and reaches a value $1.2 \times 10^{-3} \Omega^{-1} \mathrm{~cm}^{-1}$ at the temperature $271.7 \mathrm{~K}$. Now it starts decreasing and a sharp fall is observed to a minimum value $4 \times 10^{-5}$ $\Omega^{-1} \mathrm{~cm}^{-1}$ of conductivity at $380 \mathrm{~K}$ temperature. Above this temperature, it again starts increasing up to the temperature $445 \mathrm{~K}$. On further increasing the temperature, the conductivity decreases up to $500 \mathrm{~K}$. 
Here, from the whole curve shown in Figure 2, it is noted that two maxima are found below the room temperature and one maximum above the room temperature. Each " $\lambda$ " type behavior is observed in the conductivity variation around temperature 120, 271.7 and $445 \mathrm{~K}$ respectively below and above the room temperature. Figure 4, shows the dependence of the logarithmic value of normalized conductivity on $1000 / T$ for the mentioned complex. Another curve is also plotted for the complex in the logarithmic value of conductivity versus 1/ $T$ and shown in Figure 5. The temperature dependence conductivity behavior is fitted theoretically and observed that a metallic type nature of the sample below the room temperature. This follows the $T^{-5}$ and $T^{-3}$ laws in the first metallic region and $T^{-5}$ in the second metallic region in the temperature ranges $120 \leq T \leq$ $160 \mathrm{~K}, 170 \leq T \leq 265 \mathrm{~K}$ and $275 \leq T \leq 300 \mathrm{~K}$ respectively. Whereas semiconducting nature of the sample at low temperatures follows $\ln \sigma$ vs $T^{-1}$ law and show different values of activation energy in different regions. We have calculated activation energy $0.078 \mathrm{eV}$ in temperature region $50 \leq T \leq 110 \mathrm{~K}$ and 0.032 $\mathrm{eV}$ in temperature region $265 \leq T \leq 270 \mathrm{~K}$. Above the room temperature, the metallic behavior of the sample follows $T^{-2}$ and $T^{-5}$ laws in temperature regions $320 \leq T \leq 375 \mathrm{~K}$ and then $450 \mathrm{~K}$ respectively. Whereas semiconducting nature of the sample above the room temperature, which is well fitted with exponential law $\ln \sigma$ vs $T^{-1}$ and gives activation energy of $0.13 \mathrm{eV}$ in temperature region $380 \leq$ $T \leq 450 \mathrm{~K}$.

\subsubsection{Single Crystal Grown by Diffusion Method}

Quadruplet, black-reddish single crystal of K-TCNQ grown by diffusion method [44] in acetonitrile have shown conductivity $\sigma_{R T} \approx 2.41 \times 10^{-4} \Omega^{-1} \mathrm{~cm}^{-1}$ at room temperature with an activation energy of $0.1 \mathrm{eV}$. This sample shows almost a similar behavior of conductivity with variation in temperature to the sample is grown by solution growth method in the range of 30 to $500 \mathrm{~K}$. On starting the measurement with increasing the temperature from $30 \mathrm{~K}$, initially the conductivity increases. It reaches a maximum value of conductivity $5.48 \times 10^{-3} \Omega^{-1} \mathrm{~cm}^{-1}$ at a temperature $120 \mathrm{~K}$. On further increasing the temperature, conductivity goes on decreasing and reaches a value $4.7 \times 10^{-4} \Omega^{-1} \mathrm{~cm}^{-1}$ at temperature $265 \mathrm{~K}$. The conductivity of the complex increasing again and reaches a value $2 \times 10^{-3}$ $\Omega^{-1} \mathrm{~cm}^{-1}$ at the temperature $271.7 \mathrm{~K}$, on further increasing the temperature. Now it starts decreasing to a minimum value $4.4 \times 10^{-5} \Omega^{-1} \mathrm{~cm}^{-1}$ of conductivity at 380 $\mathrm{K}$ temperature. Above this temperature, it again starts to increase up to the temperature $445 \mathrm{~K}$ and reaches a value $1.8 \times 10^{-3} \Omega^{-1} \mathrm{~cm}^{-1}$. On further increasing the temperature, the conductivity decreases up to $500 \mathrm{~K}$.

The temperature dependent electrical behavior of the studied sample is shown in Figure 3. Two maxima below the room temperature and one maximum are found above the room temperature. A " $\lambda$ " type behavior is observed in the conductivity variation around temperature 120, 271.7 and $445 \mathrm{~K}$ respectively below and above the room temperature. Figure 4 and Figure 5, show the plot of the natural logarithmic value of normalized conductivity versus $1000 / \mathrm{T}$ and natural logarithmic values of conductivity versus $1 / T$. 


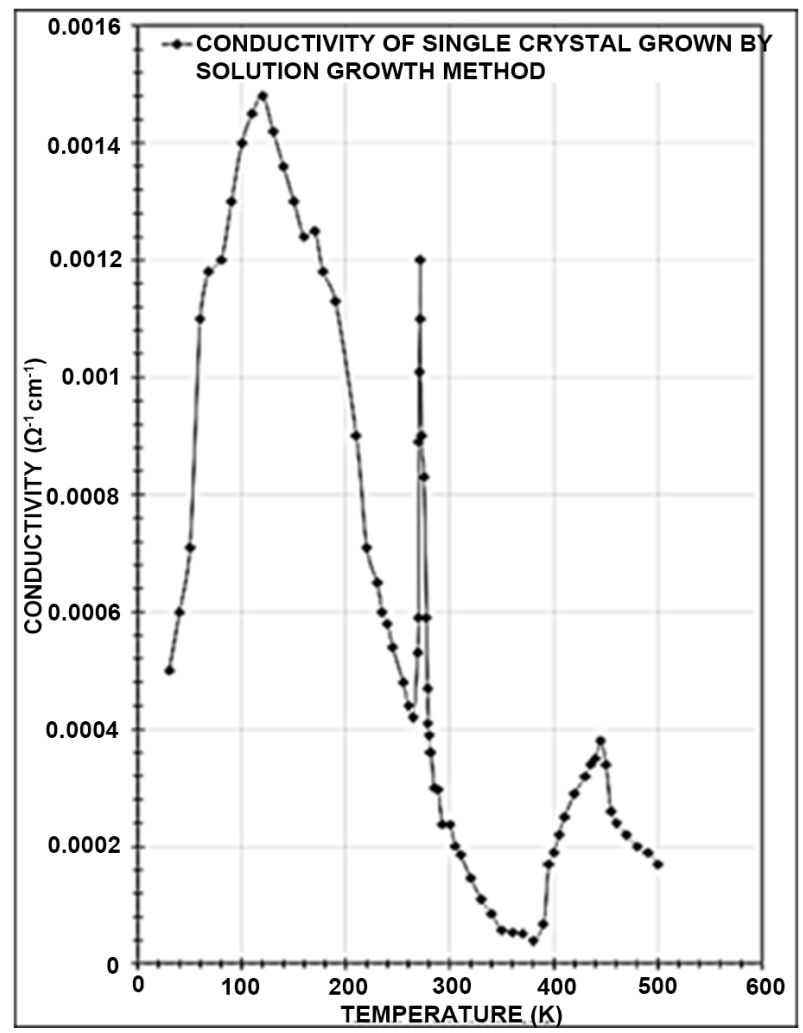

Figure 2. Plot of conductivity as a function of temperature in the region 30 to $500 \mathrm{~K}$ for K-TCNQ single crystal grown by solution growth method.

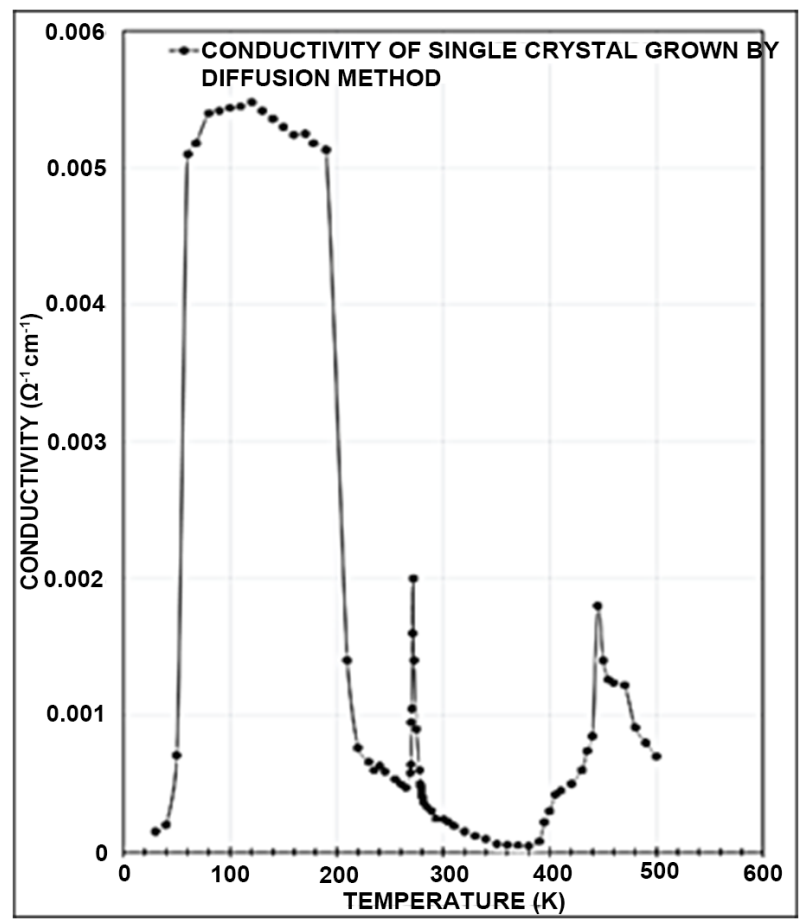

Figure 3. Plot of conductivity as a function of temperature in the region 30 to $500 \mathrm{~K}$ for K-TCNQ single crystal grown by diffusion method. 


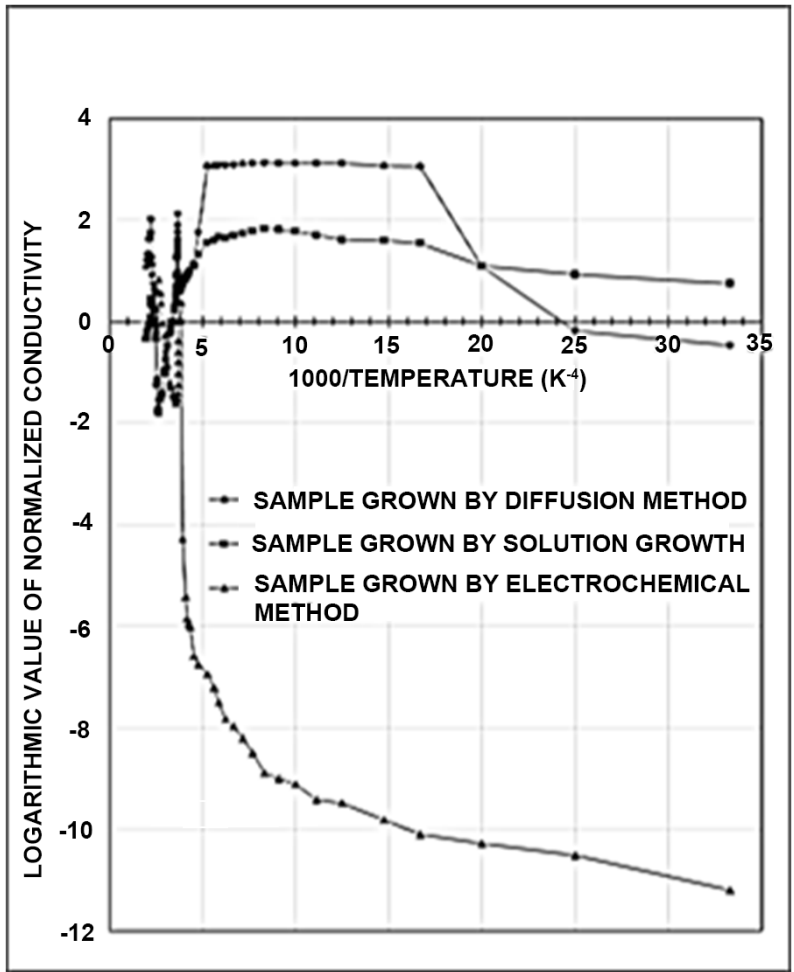

Figure 4. Plot of logarithmic value of normalized conductivity as a function of 1000/temperature in the region 30 to 500 $\mathrm{K}$ for K-TCNQ single crystal grown by diffusion method.

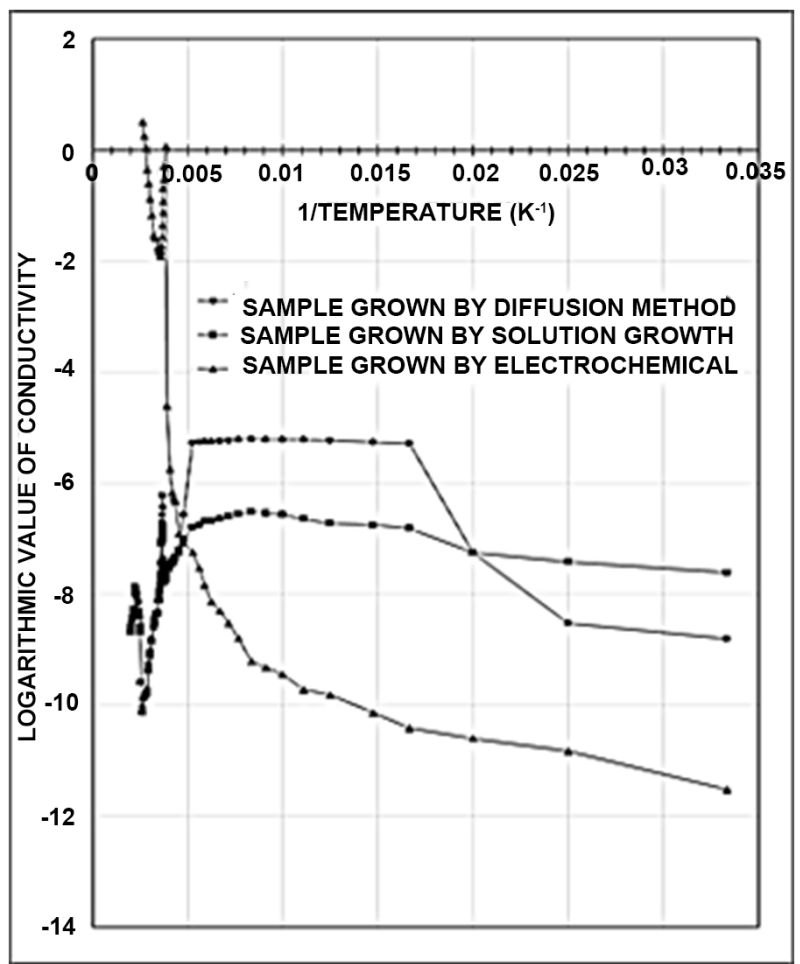

Figure 5. Plot of logarithmic value of conductivity against $1 /$ temperature in the region 30 to $500 \mathrm{~K}$ for K-TCNQ single crystal grown by diffusion method. 
The observed behavior of the sample is fitted theoretically and we found a metallic type nature of the sample below the room temperature. This follows the $T^{-5}$ and $T^{-3}$ laws in the first metallic region and $T^{-5}$ in the second metallic region in the temperature ranges $120 \leq T \leq 160 \mathrm{~K}, 170 \leq T \leq 190 \mathrm{~K}, 200 \leq T \leq 265 \mathrm{~K}$ and $275 \leq T \leq 300 \mathrm{~K}$ respectively. Whereas semiconducting nature of the sample at low temperatures follows $\ln \sigma$ vs $T^{-1}$ law and shows different values of activation energy in different regions as indicated by Figure 5 . We have calculated activation energy $0.076 \mathrm{eV}$ in temperature region $50 \leq T \leq 110 \mathrm{~K}$ and $0.030 \mathrm{eV}$ in temperature region $265 \leq T \leq 270 \mathrm{~K}$.

Above the room temperature, the metallic behavior of the sample follows $T^{-2}$ and $T^{-5}$ laws in temperature regions $320 \leq T \leq 375 \mathrm{~K}$ and then $450 \mathrm{~K}$ respectively. Whereas semiconducting nature of the sample above the room temperature, which is well agreed with exponential law $\ln \sigma$ vs $T^{-1}$ and gives activation energy of $0.12 \mathrm{eV}$ in temperature region $380 \leq T \leq 450 \mathrm{~K}$.

\section{Discussion}

In the preceding section, the detailed observations of temperature dependence electrical conductivity in the wide temperature range from 30 to $500 \mathrm{~K}$ of three $\mathrm{K}-\mathrm{TCNQ}$ samples grown by different methods were presented. It is notable here that K-TCNQ is a RIS with symmetric donor molecules. It has nonequivalent molecular stacking. During this study, we have observed a number of peculiar features as mentioned below, to be discussed which show a typical behavior of this complex.

1) It is observed from the literature that the RIS crystals with asymmetric donor molecules like Qn-TCNQ2 and Ad-TCNQ2 have a large conductivity than those with symmetric donor molecules like K-TCNQ, Li-TCNQ and Rb-TCNQ etc.

2) In the present study, the crystals grown by the electrochemical method have higher conductivity than those of the same salt grown by other methods.

3) More than one phase transitions are observed in the samples grown by solution growth and diffusion methods.

4) The conductivity follows the T- $\gamma$ law in the different temperature regions, where the samples are showing metallic type behavior. The value of $\gamma$ is an integer and depends upon the nature of scattering involved.

5) Activation energy changes with temperature, where the samples show semiconducting behavior.

A possible explanation is given as follows for the above-mentioned features of the studied samples on the basis of the analysis in the framework of their molecular structure, disorder effect, impurity effect, Coulomb interaction, charge density waves (CDW) effect, scattering effect and 3-D effect etc.

\subsection{Larger Conductivity of Crystals with Asymmetric Donor Molecules}

It is evident that reasonable high conductivity of these organic complexes arises 
from the overlap of the molecular orbitals. This caused delocalization of the $\pi$-electrons along the axis of stacking. Since the difference between the ionization potential of donor and electron affinity of the acceptor is fairly large in K-TCNQ [45], as a result these compounds have a large band gap, therefore a low conductivity. The second evidence is also important here which correlate the electrical properties of the salts to the intramolecular donor properties [46]. It states that donors with large dipole moment are arranged regularly leading to a charge-ordered state with low conductivity and a large energy gap. On the other hand, $\mathrm{Qn}-\mathrm{TCNQ}_{2}$ typed complexes with smaller donor dipoles are stacked randomly. When inter-chain and intra-chain coulomb forces are much larger than the random donor-acceptor interactions, the materials have a small but well-defined energy gap. It is believed that K-TCNQ is ordered and donor dipoles are alternating in this salt. Whereas the salts $\mathrm{Qn}-\mathrm{TCNQ}_{2}$ and $\mathrm{Ad}-\mathrm{TCNQ} \mathrm{C}_{2}$ represent another class of material with smaller donor dipoles stacked randomly [46]. Thus, without going into much of the details the first observation is justified that the salts with asymmetric donor molecules have a large conductivity than those with the symmetric donor molecules.

\subsection{High Conductive Crystals Grown by Electrochemical Method}

In order to explain the effect of methods of growing of these salts on their conductivity, it has been considered and discussed by Kamras et al. [39] Variation in conductivity depending on the method of sample preparation is correlated with the presence of ionized $\mathrm{TCNQ}^{-}$in the samples. Increasing concentration of the ionized $\mathrm{TCNQ}^{-}$is resulted in decreasing conductivity. It is concluded that impurities act as an excess potential and overall effect is a reduction of the conductivity. This reduction depends on the strength and distribution of the impurity potential. In all the studied complexes grown in different ways than electrochemical method, a weak band at $20,000 \mathrm{~cm}^{-1}$ in optical absorption spectra is observed. The position of this band agrees with that found by Kammandeur et al. [47] for ionized $\mathrm{TCNQ}^{-}$. Therefore, it is believed that this observation indicates the presence of $\mathrm{TCNQ}^{-}$ions. The comparison of $\mathrm{TCNQ}^{0}$ and $\mathrm{TCNQ}^{-}$are confirmed from the standard chemical analysis and optical absorption spectra [41]. Increasing $\mathrm{TCNQ}^{-}$concentration results in an increase of $\mathrm{D}^{+}$(donor) concentration to maintain the neutrality of the crystal. The localized $\mathrm{D}^{+}$impurities may then be responsible for the drastic variation in conductivity. It is believed that the crystals grown by the electrochemical method are purer than grown by other methods. This has also pointed out by Williams et al. [48] in his previous study. Therefore, the nature of variation of conductivity is different in the samples grown by different methods for the same salt, as well as the conductivity is higher in the sample grown by electrochemical method.

\subsection{Multi Phases}

The third observed fact can be explained on the basis of the earlier reported stu- 
dies based on various effects like of an important role of impurities [49], the dominant role of electron-electron interaction [50], the temperature dependent mobility [51] etc. Some other workers have emphasized on the role of disorder [46] also. Gogolin [52] and other co-workers have discussed phase transition in the framework of a 1-D disordered metal model where the random potential is larger than both the on-chain and inter-chain correlation energies. Kamras [39] and others have explained the observed phase transitions on the basis of the effect of impurities. However, it may mention here that transitions obtained in many such cases may be due to the incorporation of acetonitrile molecules in the lattice, used as a solvent for the synthesis of samples [53].

It is observed that four metal to semiconductor phase transitions at certain temperatures in the temperature range from 30 to $500 \mathrm{~K}$ in K-TCNQ salt grown by solution growth and diffusion methods. These are found at 120, 270, 380 and $470 \mathrm{~K}$ respectively, whereas only two phase transitions are observed in the salt grown by electrochemical method. These are observed around 270 and $380 \mathrm{~K}$ respectively. The conductivity at room temperature of the crystals grown by electrochemically is more in magnitude by an order of 3 than those grown by other methods.

Table 1 shows a comparative study of electrical conductivity at room temperature, transition temperatures and activation energy of K-TCNQ reported earlier in different studies and the present work. As mentioned in Table 1, Konno et al. [54] found that K-TCNQ undergoes a phase transition around $395 \mathrm{~K}$ having conductivity $3.5 \times 10^{-4} \Omega^{-1} \mathrm{~cm}^{-1}$ at room temperature, whereas Khanna et al. [55] have obtained the value of conductivity for this complex grown by U-tube diffusion method in the same order $1 \times 10^{-4} \Omega^{-1} \mathrm{~cm}^{-1}$ at room temperature with a magnetic transition near $400 \mathrm{~K}$. Sakai et al. [56] have also reported the synthesis of K-TCNQ and Rb-TCNQ crystals by the diffusion method. They found the conductivity of K-TCNQ crystals of the order of $10^{-4}$ at room temperature and phase transition at $391 \pm 6 \mathrm{~K}$. Vegter et al. [12] have reported a phase transition at $381 \mathrm{~K}$ for the reddish purple K-TCNQ. A high-pressure electrical resistivity study is also performed on the powder of K-TCNQ sample [56] and it was found that different samples exhibit different peculiar behavior in the pressure-dependent resistivity measurements. The explanation for this was given in terms of chemical impurities and some lattice defects. It is possible that each crystal may have a different imperfection synthesized by diffusion and crystal growth methods.

The occurrence of more phase transitions in these samples can be explained on the basis of the two types of independent rows of $\mathrm{TCNQ}^{-1}$, existing simultaneously in single crystals of K-TCNQ at room temperature. It is suggested by Troung et al. [57] that K-TCNQ crystal lattice has monoclinic structure with two space groups $P_{1}^{2} / n$ and $P_{1}^{2} / c$, where the TCNQ molecules form charge resonance bounded rows along the a-axis. The interaction between the rows is weak. Within a row interplanar spacing of about 3.1 and $3.5 \mathrm{~A}$ alternates, that is 
dimerization of molecule or diatic structure in the low-temperature phase. Where the one-dimensional unit cell has two molecules and the lattice constant for the a-direction becomes twice that of the high-temperature phase. This means the alkali-TCNQ salts are pseudo-one-dimensional salt for the a-direction [56]. On the other hand, the dimerization of TCNQ is not present above the transition temperature, where one-dimensional unit cell has one molecule only as suggested by Tanner [58]. Therefore, one of the phase transitions in alkali-TCNQ salts may be discussed in terms of a Peierls transition. It is also notable that some of the possible low conducting RIS salts have polymorphism characteristics and iso-structural series. The different crystalline forms and different iso-structural complexes may show very different behavior; in particular for the electrical conductivity. K-TCNQ is also having polymorphism characteristics and thus shows anomalous electrical conductivity behavior [59].

Microscopically, the occurrence of four transitions in our samples can be understood on the basis of the anti-ferromagnetism, Peierls instability, charge density waves and Coulomb repulsion. These all phenomena play very important roles in the peculiar electrical and magnetic properties. The phase transition observed at $370 \mathrm{~K}$ may be associated with anti-ferromagnetic phase transition, which is reported by others at $390 \pm 6 \mathrm{~K}$. However transition temperature, in this case, is less than that of others, but more purity of the crystals may be the cause. Here the sample may behave like a spin Peierls system and show anomalies in magnetic susceptibility measurements as reported for other K-TCNQ samples by Khanna et al. [55]

The other transitions appeared at 270 and $470 \mathrm{~K}$ may be due to Peierls instability and associated $\mathrm{CDW}$ occurring for two types of $\mathrm{TCNQ}^{-}$chains at different temperatures. The giant anomalies obtained in conductivity can then be explained in terms of the Fermi surface fraction destroyed by the formation of independent CDWs as in other CDW systems [60]. Coulomb repulsive interactions may also play an important role at low temperature giving rise to phase transition at $120 \mathrm{~K}$. However, these explanations need further confirmation by other more advanced experimental studies.

\subsection{Conductivity Follows Different Laws}

Below and above the room temperature, the logarithmic value of conductivity part below the transition in certain temperature ranges in which the compound shows semiconducting behavior is theoretically well fitted with $\ln \sigma$ vs $T^{-1}$ law and K-TCNQ gives a linear relation in these regions as shown in Figure 5, which indicates its behavior like a typical intrinsic semiconductor.

It is suggested by Bloch et al. [38] [61] that the part of the conductivity above the transition temperature and showing metallic behavior is theoretically fitted with the law $\sigma$ vs $T^{-\gamma}$, where $\gamma$ has different values like 1,2, 3 and 5. This feature of the K-TCNQ samples can be ascribed on the basis of different scattering process involved. 
The theory of temperature dependent electrical properties of metal, at high temperatures $\left(T \gg \theta_{D}\right.$ ), the total number of phonons on the surface of allowed wave vectors for scattering of a given electron is directly proportional to $T$ and the number of scatters increases linearly with $T$, hence

$$
\sigma \propto T^{-1} \text { Where, } T \gg \theta_{D}
$$

Thus, quite away from the transition temperature ( $T$ high) or in the regions where conductivity falls gradually with increase in temperature, the behavior is well fitted with the $T^{-1}$ law. For well below $\theta_{D}$ it is found that the net electron-phonon scattering rate declines as $T^{3}$, and hence

$$
\sigma \propto T^{-3} \text { Where, } T \ll \theta_{D}
$$

However, an additional factor of $T^{2}$, expressing the growing predominance of forwarding scattering with decline temperature comes into the picture in metals. This is true even in anisotropic metals like as here in the case of 1-D complexes. When combined with the $T^{3}$ dependence of the scattering as mentioned above, it leads the Bloch $T^{5}$ law, where

$$
\sigma \propto T^{-5} \text { Where, } T \ll \theta_{D}
$$

Thus, we observed that near the transition temperature or where the fall of conductivity is sharp with temperature, the behavior is well fitted with the $T^{-5}$ law. In the same regions, the experimental data fit well with the $T^{+3}$ law as mentioned above. Further, the $T^{-5}$ dependence of conductivity is usually masked by defects and impurities. Thus it would be observed in more pure samples. The $T^{2}$ dependence of conductivity in certain temperature regions appears as a transition from $T^{-5}$ to $T^{-1}$ dependence with an increase in temperature. However, it may appear independently also if electron-phonon interaction term is included in the Hubbard model which takes into account the effect of electronic correlations [62] [63]. Similarly, the $T^{-1}$ dependence of conductivity may also arise independently due to electron scattering process in the Hubbard model [64].

\subsection{Variation in Activation Energy}

Different laws are followed by the studied material in the different temperature regions. The activation energy is a function of temperature. Therefore a variation is observed in the activation energy and it is increased with decreasing temperature in the regions where the sample is showing semiconducting behavior. The increase in the activation energy below $T_{c}$ indicates the appearance of the dimerization gap.

\section{Conclusions}

The results of this study and some of the earlier reported are summarized in Table 1. This comparison markedly indicates the high purity and much higher electrical conductivity of single crystals grown by an electrochemical method in this case. Previously electrical conductivity was observed for this complex in the 
range of $10^{-7}$ to $10^{-4} \Omega^{-1} \mathrm{~cm}^{-1}$, whereas in our case it is of the order of $10^{-1}$ $\Omega^{-1} \mathrm{~cm}^{-1}$.

It is also indicated in Table 1 that the activation energy is less of the order of one than the others obtained at room temperature. Activation energy changes for different semiconducting regions observed at certainly different temperatures, even for the same sample.

Some unusual metal to insulator phase transitions, which are not reported earlier, are also observed in the studied samples. It is also noticeable from the present study that some small jumps in electrical conductivity are observed at certain temperatures in some of the samples. The crystals grown of K-TCNQ are fragile and thin also. When the temperature dependent conductivity measurements are performed on these crystals, some small cracks may be developed inside the crystals due to the variation of temperature and current. This may be one of the reasons behind the small jumps in the conductivity.

Overall it is concluded that the samples of K-TCNQ grown by different methods are showing some very interesting behavior and conspicuous phase transitions in temperature dependence conductivity study. Those can be understood principally by disorder and impurity effects at low temperature, scattering due to impurities and electron-phonon scattering at higher temperatures. However, an advanced investigation and precise evaluation will be required for the better understanding of the electrical transport properties of K-TCNQ salt. It is also suggested that due to non-linearity and switching behavior of the complex, a wide range of applicability should be tested in the direction of nonvolatile memories and ultra-high density data storage etc.

\section{Acknowledgements}

The author gratefully acknowledges the financial support provided by Department of Science and Technology (DST), India and thanks to Prof. M. L. Kalra for the useful discussion during the work.

\section{References}

[1] Canevet, D., Salle, M., Zhang, G., Zhang, D. and Zhu, D. (2009) Tetrathiafulvalene (TTF) Derivatives: Key Building-Blocks for Switchable Processes. Chemical Communications, 17, 2245-2269. https://doi.org/10.1039/b818607n

[2] Singleton, J. (2002) Why Do Physicists Love Charge-Transfer Salts? Journal of Solid State Chemistry, 168, 675-689. https://doi.org/10.1006/jssc.2002.9766

[3] Devic, T., Evain, M., Moelo, Y., Canadell, E., Auban-Senzier, P., Fourmigue, M. and Batail, P. (2003) Single Crystalline Commensurate Metallic Assemblages of $\pi$-slabs and $\mathrm{CdI}_{2}$-Type Layers: Synthesis and Properties of $\beta$-(EDT-TTF- $\left.\mathrm{I}_{2}\right)_{2}\left[\mathrm{~Pb}_{5 / 61 / 6} \mathrm{I}_{2}\right]_{3}$ and $\beta$-(EDT-TTF- $\left.\mathrm{I}_{2}\right)_{2}\left[\mathrm{~Pb}_{2 / 3+x} \mathrm{Ag}_{1 / 3-2 x x} \mathrm{I}_{2}\right]_{3}, x=0.05$. Journal of the American Chemical Society, 125, 3295-3301. https://doi.org/10.1021/ja0290431

[4] Suzuki, W., Fujiwara, E., Kobayashi, A., Fujishiro, Y., Nishibori, E., Takata, M., Sakata, M., Fujiwara, H. and Kobayashi, H. (2003) Highly Conducting Crystals Based on Single-Component Gold Complexes with Extended-TTF Dithiolate Ligands. Journal of the American Chemical Society, 125, 1486-1487. 
https://doi.org/10.1021/ja0292243

[5] Yamada, J.I., Toita, T., Akutsu, H., Nakatsuji, S., Nishikawa, H., Ikemoto, I., Kikuchi, K., Choi, E.S., Graf, D. and Brooks, J.S. (2003) A New Organic Superconductor, $\beta$-(BDA-TTP) ${ }_{2} \mathrm{GaCl}_{4} \quad[\mathrm{BDA}-\mathrm{TTP}=2,5$-(1,3-dithian-2-ylidene)-1,3,4,6-tetrathiapentalene]. Chemical Communications, 2230-2231. https://doi.org/10.1039/B306314C

[6] Melby, L.R., Harder, R.J., Hertler, W.R., Mahler, W., Benson, R.E. and Mochel, W.E. (1962) Substituted Quinodimethans. II. Anion-Radical Derivatives and Complexes of 7,7,8,8-Tetracyanoquinodimethan. Journal of the American Chemical Society, 84, 3370-3374. https://doi.org/10.1021/ja00876a028

[7] Garito, A.F. and Heeger, A.J. (1974) Design and Synthesis of Organic Metals. Accounts of Chemical Research, 7, 232-240. https://doi.org/10.1021/ar50079a004

[8] Peristein, J.H. (1977) “Organic Metals"-The Intermolecular Migration of Aromaticity. Angewandte Chemie International Edition, 16, 519-534. https://doi.org/10.1002/anie.197705191

[9] Wheland, R.C. and Gillson, J.L. (1976) Synthesis of Electrically Conductive Organic Solids. Journal of the American Chemical Society, 98, 3916-3925.

https://doi.org/10.1021/ja00429a030

[10] Vlasova, R.M., Gutman, A.I., Kuzina, V.V. and Sherle, A.I. (1970) Investigation of the Complex Conductivity of Ion-Radical Salts of Tetracynoquinodimethane. Soviet Physics, Solid State, 12, 2979.

[11] Afify, H.H., Abdel-Kerim, F.M., Aly, H.F. and Shabaka, A.A. (1978) The Electrical Conductance of Some Alkali- and Divalent Transition Metal TCNQ Salts. Zeitschrift für Naturforschung, 33a, 344-346. https://doi.org/10.1515/zna-1978-0312

[12] Vegter, J.G., Hibma, T. and Kommandeur, J. (1969) New Phase Transitions in Simple M-TCNQ-Salts. Chemical Physics Letters, 3, 427-429. https://doi.org/10.1016/0009-2614(69)80157-0

[13] Jerome, D. and Schulz, H.J. (2002) Organic Conductors and Superconductors. Advance in Physics, 51, 293-479. https://doi.org/10.1080/00018730110116362

[14] Xu, W., Chen, G.R., Li, R.J. and Hua, Z.Y. (1995) Two New All-Organic Complexes with Electrical Bistable States. Applied Physics Letters, 67, 2241-2242. https://doi.org/10.1063/1.115116

[15] Oyamada, T., Tanaka, H., Matsushige, K., Sababe, H. and Adachi, C. (2003) Switching Effect in Cu:TCNQ Charge Transfer-Complex Thin Films by Vacuum Codeposition. Applied Physics Letters, 83, 2241. https://doi.org/10.1063/1.1600848

[16] Muller, R., Jonge, S. De, Myny, K., Wouters, D.J., Geone, J. and Heremans, P. (2006) Organic CuTCNQ Integrated in Complementary Metal Oxide Semiconductor Copper Back-End-of-Line for Nonvolatile Memories. Applied Physics Letters, 89, Article ID: 223501. https://doi.org/10.1063/1.2388883

[17] Ran, C.B., Peng, H.L., Zhou, W., Yu, X.C. and Liu, Z.F. (2005) Thermochemical Hole Burning on a Series of N-Substituted Morpholinium 7,7,8,8-Tetracyanoquinodimethane Charge-Transfer Complexes for Data Storage. The Journal of Physical Chemistry B, 109, 22486-22490. https://doi.org/10.1021/jp0528616

[18] Yu, X.C., Peng, H.L., Ran, C.B., Sun, L., Zhang, R. and Liu, Z.F. (2005) Scanning Tunneling Microscope-Based Thermochemical Hole Burning on a Series of Charge Transfer Complexes. Applied Physics Letters, 86, Article ID: 133105. https://doi.org/10.1063/1.1883315

[19] Hiroka, M., Hasegawa, T., Abe, Y., Yamada, T., Tokura, Y., Yamochi, H., Saito, G., Akutagawa, T. and Nakamura, T. (2006) Ink-Jet Printing of Organic Metal Elec- 
trodes Using Charge-Transfer Compounds. Applied Physics Letters, 89, Article ID: 173504.

[20] Blochwitz, J., Pfeiffer, M., Fritz, T. and Leo, K. (1998) Low Voltage Organic Light Emitting Diodes Featuring Doped Phthalocyanine as Hole Transport Material. Applied Physics Letters, 73, 729. https://doi.org/10.1063/1.121982

[21] Law, C.W., Lau, K.M., Fung, M.K., Chan, M.Y., Wong, F.L., Lee, C.S. and Lee, S.T. (2006) Effective Organic-Based Connection Unit for Stacked Organic Light-Emitting Devices. Applied Physics Letters, 89, Article ID: 133511. https://doi.org/10.1063/1.2357846

[22] Sakai, M., Iizuka, M., Nakamura, M. and Kudo, K. (2005) Organic Nano-Transistor Fabricated by Co-Evaporation Method under Alternating Electric Field. Synthetic Metals, 153, 293-296. https://doi.org/10.1016/j.synthmet.2005.07.156

[23] Di, C.A., Yu, G., Liu, Y.Q., Xu, X.J., Wei, D.C., Song, Y.B., Sun, Y.M., Wang, Y., Zhu, D.B., Liu, J., Liu, X.Y. and Wu, D.X. (2006) High-Performance Low-Cost Organic Field-Effect Transistors with Chemically Modified Bottom Electrodes. Journal of the American Chemical Society, 128, 16418-16419. https://doi.org/10.1021/ja066092v

[24] Liu, H.B., Zhao, Q., Li, Y.L., Liu, Y.Q., Lu, F.S., Zhuang, J.P., Wang, S., Jiang, L., Zhu, D.B., Yu, D.P. and Chi, L.F. (2005) Field Emission Properties of Large-Area Nanowires of Organic Charge-Transfer Complexes. Journal of the American Chemical Society, 127, 1120-1121. https://doi.org/10.1021/ja0438359

[25] Rosenfeld, S.M., Lawler, R.G. and Ward, H.R. (1973) Photo-CIDNP [Chemically Induced Dynamic Nuclear Polarization] Detection of Transient Intermediates. Enol of Acetophenone. Journal of the American Chemical Society, 95, 946-948. https://doi.org/10.1021/ja00784a065

[26] Abokowitz, A., Epstein, A.J., Griffiths, C.H., Miller, J.S. and Slade, M.L. (1977) Characterization of Trimethylammonium Iodide and 7,7,8,8-Tetracyano-p-Quinodimethane Reaction Product. A One-Dimensional "Metal-Like" Complex. Journal of the American Chemical Society, 99, 5304-5307. https://doi.org/10.1021/ja00458a013

[27] Zhang, H.Q. (2004) The Study on the Peierls Phase Transition of One-Dimensional Organic Conductors. Acta Physica Sinica, 53, 1162-1165. (In Chinese) http://wulixb.iphy.ac.cn/CN/Y2004/V53/I4/1162

[28] Zhang, H.Q., Liu, S.J. and Li, R.W. (2005) The Study on the Peierls Phase Transition of TTF-TCNQ. Acta Physica Sinica, 54, 3317-3320. (In Chinese) http://wulixb.iphy.ac.cn/CN/Y2005/V54/I7/3317

[29] Iwasa, Y., Mizuhashi, K., Koda, T., Tokura, Y. and Saito, G. (1994) Metal-Insulator Transition and Antiferromagnetic Order in Bis(Ethylenedithio)Tetrathiafulvalene Tetracyanoquinodimethane (BEDT-TTF) (TCNQ). Physical Review B, 49, 3580. https://doi.org/10.1103/PhysRevB.49.3580

[30] Hiraki, K., Furuta, S., Takahashi, T., Kondo, R., Kagoshima, S., Hasegawa, T., Mochida, T. and Iwasa, Y. (2002) Metal-Insulator Transition of Donor-Acceptor-Type Organic Charge-Transfer Complex (BETS $)_{2}\left(\mathrm{Br}_{2} \mathrm{TCNQ}\right)$ : Site-Selective NMR Measurements. Physical Review B, 66, Article ID: 35104.

https://doi.org/10.1103/PhysRevB.66.035104

[31] Taniguchi, M., Misaki, Y., Yamabe, T., Tanaka, K., Murata, K. and Mori, T. (1999) (CPDT-STF) (TCNQ): A New Charge-Transfer Complex Metallic Down to Low Temperature. Solid State Communications, 111, 559-564. https://doi.org/10.1016/S0038-1098(99)00247-1

[32] Bartholin, H., Baudour, J.L., Breandon, C., Tchapoutian, R., Cailleau, H. and Perrin, 
D. (1987) A Possible Devil's Staircase in TTF-Chloranil at the Neutral-Ionic Transition Observed by Electric Conductivity Measurements. Solid State Communications, 63, 223-225. https://doi.org/10.1016/0038-1098(87)90845-3

[33] Buron-Le, M.C., Lemee-Cailleau, M.H., Cailleau, H., Toudic, B., Moreac, A., Moussa, F., Ayache, C. and Karl, N. (2003) Thermal Hysteresis Phenomena and Mesoscopic Phase Coexistence around the Neutral-Ionic Phase Transition in TTF-CA and TMB-TCNQ. Physical Review B, 68, Article ID: 64103. https://doi.org/10.1103/PhysRevB.68.064103

[34] Le Cointe, M., Lemee-Cailleau, M.H., Cailleau, H., Toudic, B., Toupet, L., Heger, G., Moussa, F., Schweiss, P., Kraft, K.H. and Karl, N. (1995) Symmetry Breaking and Structural Changes at the Neutral-to-Ionic Transition in Tetrathiafulvalene- $p$ Chloranil. Physical Review B, 51, 3374. https://doi.org/10.1103/PhysRevB.51.3374

[35] Soos, Z.G., Bewick, S.A., Painelli, A. and Girlando, A. (2005) Electronic and Structural Instabilities of Mixed-Stack Organic Charge-Transfer Salts. Synthetic Metals, 155, 357-364. https://doi.org/10.1016/j.synthmet.2005.09.013

[36] Lumsden, M.D. and Gaulin, B.D. (1999) Critical Phenomena at the Spin-Peierls Transition in MEM (TCNQ) $)_{2}$. Physical Review B, 59, 9372.

https://doi.org/10.1103/PhysRevB.59.9372

[37] Herbstein, F.H. (2006) On the Mechanism of Some First-Order Enantiotropic Solid-State Phase Transitions: From Simon through Ubbelohde to Mnyukh. Acta Crystallographica Section B, 62, 341-383. https://doi.org/10.1107/S0108768106008640

[38] Bloch, A.N., Weisman, R.B. and Varma, C.M. (1972) Identification of a Class of Disordered One-Dimensional Conductors. Physical Review Letters, 28, 753. https://doi.org/10.1103/PhysRevLett.28.753

[39] Kamaras, K., Ritvay, K.E., Mihaly, G., Gruner, G. and Rysaba, N. (1977) Impurity Effects in the Organic Charge Transfer Salt Qn(TCNQ) 2 . Journal of Physics C: Solid State Physics, 10, L423. https://doi.org/10.1088/0022-3719/10/15/006

[40] Cowan, D., Shu, P., Hu, C., Krug, W., Carruthers, T., Poehler, T. and Bloch, A.N. (1974) Energy and Charge Transfer in Organic Semiconductors. Ed. by K. Masuda and M. Silver, Plenum, New York, 25.

[41] Singh, Y., Goswami, D.P., Bala, M. and Kalra, M.L. (1992) Large Needle-Shaped Highly Conducting Organic Charge Transfer Complexes with Asymmetric Donor Molecules. Journal of Crystal Growth, 123, 601-604. https://doi.org/10.1016/0022-0248(92)90623-Q

[42] Kaplan, M.L. (1976) A Three-Chamber Diffusion Apparatus for the Growth of Single Crystals of Organic Donor-Acceptor Salts. Journal of Crystal Growth, 33, 161-164. https://doi.org/10.1016/0022-0248(76)90093-2

[43] Anzai, H. (1976) Growth of Large-Crystals of Charge-Transfer Complex, Tetrathiafulvalene-Tetracyanoquinodimethane (TTF-TCNQ). Journal of Crystal Growth, 33, 185-187. https://doi.org/10.1016/0022-0248(76)90100-7

[44] Singh, Y. (2013) Electrical Resistivity Measurements: A Review. International Journal of Modern Physics. Conference Series, 22, 745-756.

[45] Andre, J.J., Bieber, A. and Gautier, F. (1976) Physical Properties of Highly Anisotropic Systems: Radical-Ion Salts and Charge Transfer Complexes. Annales de Physique, 1, 145-256.

[46] Holczer, K., Mihaly, G., Jannossy, A., Gruner, G. and Kertesz, M. (1978) Complex TCNQ Salts with Asymmetric Donors. I. Transport Properties. Journal of Physics C: Solid State Physics, 11, 4707. https://doi.org/10.1088/0022-3719/11/23/016 
[47] Jonkman, H.T. and Kommandeur, J. (1972) The UV Spectra and Their Calculation of TCNQ and Its Mono- and Di-Valent Anion. Chemical Physics Letters, 15, 496-499. https://doi.org/10.1016/0009-2614(72)80357-9

[48] Williams, J.M., Wang, H.H., Emge, T.J., Geiser, U., Beno, M.A., Leung, P.C.W., Douglas Carlson, K., Thorn, R.J., Schultz, A.J. and Whangbo, M.-H. (1987) Rational Design of Synthetic Metal Superconductors. In: Lippard, S.J., Ed., Progress in Inorganic Chemistry, Vol. 35, John Wiley \& Sons, Inc., Hoboken, NJ, 80.

[49] Abrikosov, A.A. and Ryzhkin, I.A. (1977) On the Theory of the Conductivity of a Quasi-One-Dimensional Metal. Solid State Communications, 24, 317-318. https://doi.org/10.1016/0038-1098(77)90216-2

[50] Kwak, I.F., Beni, G. and Chaikin, P.M. (1976) Thermoelectric Power in HubbardModel Systems with Different Densities: $N$-Methylphenazinium-Tetracyanoquinodimethane (NMP-TCNQ), and Quinolinium Ditetracyanoquinodimethane. Physical Review B, 13, 641. https://doi.org/10.1103/PhysRevB.13.641

[51] Epstein, A.J. and Conwell, E.M. (1977) Model for Conduction in TCNQ Salts. Solid State Communications, 24, 627-630. https://doi.org/10.1016/0038-1098(77)90377-5

[52] Gogolin, A.A., Mel'nikov, V.I. and Rashba, E.I. (1975) Conductivity in a Disordered One-Dimensional System Induced by Electron-Phonon Interaction. Zhurnal Ėksperimental'noi i Teoreticheskoi Fiziki, 69, 327-349.

[53] Ashwell, G.J., Sandy, I.M., Chyla, A. and Cross, G.H. (1987) An Alternative Explanation of the Broad Conductivity Maxima of Metalic TCNQ Salts Obstained from Acetonitrile Solution. Synthetic Metals, 19, 463-468.

https://doi.org/10.1016/0379-6779(87)90398-5

[54] Konno, M., Isihi, T. and Saito, Y. (1977) The Crystal Structures of the Low- and High-Temperature Modifications of Potassium 7,7,8,8-Tetracyanoquinodimethanide. Acta Crystallographica Section B, 33, 763-770. https://doi.org/10.1107/S0567740877004609

[55] Khanna, S.K., Bright, A.A., Garito, A.F. and Heeger, A.J. (1974) Evidence for Strong Coulomb Interactions in Alkali-TCNQ (Tetracyanoquinodimethan) Salts. Physical Review B, 10, 2139. https://doi.org/10.1103/PhysRevB.10.2139

[56] Sakai, N., Shirotani, I. and Minomura, S. (1972) Phase Transition of Alkali Metal Cation-TCNQ Anion Radical Simple Salts. Bulletin of the Chemical Society of Japan, 45, 3321-3328. https://doi.org/10.1246/bcsj.45.3321

[57] Truong, K.D. and Carlone, C. (1979) Raman Spectrum of Potassium Tetracyanoquinodimethane Crystals. Physical Review B, 20, 2238. https://doi.org/10.1103/PhysRevB.20.2238

[58] Tanner, D.B., Jacobsen, C.S., Bright, A.A. and Heeger, A.J. (1977) Infrared Studies of the Energy Gap and Electron-Phonon Interaction in Potassium-Tetracyanoquinodimethane (K-TCNQ). Physical Review B, 16, 3283. https://doi.org/10.1103/PhysRevB.16.3283

[59] Andre, J.J., Bieber, A. and Gautier, F. (1976) Physical Properties of Highly Anisotropic Systems: Radical-Ion Salts and Charge Transfer Complexes. Annales de Physique, 1, 145-256. https://doi.org/10.1051/anphys/197601040145

[60] Ong, N.P. and Monceau, P. (1977) Anomalous Transport Properties of a Linear-Chain Metal: $\mathrm{NbSe}_{3}$. Physical Review B, 16, 3443. https://doi.org/10.1103/PhysRevB.16.3443

[61] Bloch, A.N. and Varma, C.M. (1973) Some Aspects of Conduction in Disordered One-Dimensional Structures. Journal of Physics C: Solid State Physics, 6, 1849. https://doi.org/10.1088/0022-3719/6/11/010 
[62] Ihle, D. (1977) Electrical Conductivity in the Hubbard Model Including Electron-Phonon Interaction. Physica Status Solidi (B), 80, 619-628. https://doi.org/10.1002/pssb.2220800225

[63] Kalra, M.L. (1987) Electrical Conductivity of Narrow Energy Band. Proceedings of the Solid State Physics Symposium, 30C, 129.

[64] Kalra, M.L. (1983) Role of the Electron Scattering Processes in the Hubbard Model in the Insulator-to-Metal Transition. Physica Status Solidi (B), 115, 575-585. https://doi.org/10.1002/pssb.2221150229

[65] Goswami, D.P. and Kalra, M.L. (1993) Nonlinear Electrical Transport in K-TCNQ at Low Temperatures. Bulletin of Materials Science, 16, 393-396. https://doi.org/10.1007/BF02759552

[66] Siemons, W.J., Bierstedt, P.E. and Kepler, R.G. (1963) Electronic Properties of a New Class of Highly Conductive Organic Solids. The Journal of Chemical Physics, 39, 3523. https://doi.org/10.1063/1.1734224 